Article

\title{
The Non-Euclidean Hydrodynamic Klein-Gordon Equation with Perturbative Self-Interacting Field
}

\author{
Piero Chiarelli ${ }^{1,2}$ \\ 1 National Council of Research of Italy, Area of Pisa, Moruzzi 1, 56124 Pisa, Italy; pchiare@ifc.cnr.it; \\ Tel.: +39-050-315-2359; Fax: +39-050-315-2166 \\ 2 Interdepartmental Center "E.Piaggio", University of Pisa, 56124 Pisa, Italy
}

Received: 31 July 2017; Accepted: 26 September 2017; Published: 4 October 2017

\begin{abstract}
In this paper the quantum hydrodynamic approach for the Klein-Gordon equation (KGE) owning a perturbative self-interaction term is developed. The generalized model to non-Euclidean space-time allows for the determination of the quantum energy impulse tensor density of mesons, for the gravitational equation of quantum mechanical systems.
\end{abstract}

Keywords: quantum hydrodynamic representation; Bhom-Madelung approach; self-interaction; Einstein equation for quantum mechanical system

\section{Introduction}

A longstanding objective of modern physics is to describe the quantum mechanics and the quantum fields in the framework of general relativity. On an ordinary scale, the two theories are decoupled and even if theoretical models are available [1-16], it not easy to find experimental confirmations.

A first physical evidence of quantum gravity (QG) comes by way of the corrections to the Newton law, derived from the Galilean limit of the theory [17-29]. The Hawking radiation of a black hole can also be justified in the frame of QG [30-38].

Moreover, given that the uncertainty principle forbids a black hole (BH) collapse [39-44] and that the quantization of a black hole requires the existence of a fundamental state with a minimum mass, the $\mathrm{BH}$ cannot have a mass smaller than that of Planck and comparable with those of elementary particles [45].

Another phenomenon connected to QG is the cosmological constant that it is needed to model the motion of galaxies on the universal scale [46-54].

If we try to define the QG equation from the minimum action principle (MAP), the quantizing action is not self-contained under renormalization, since loop diagrams generate terms not present in the initial expression [55-59].

In the present work the author makes use of the hydrodynamic quantum description (HQD) [60-68] that has the advantage of being embedded in a classical-like formalism, where the quantization is implicitly contained into the equation of evolution [68], for deriving a gravity equation for quantum mechanical systems.

The quantum hydrodynamic approach, firstly developed by Madelung [62], describes the evolution of the complex wavefunction $\psi=|\psi| \exp \frac{i}{\hbar} S$ as a function of the two real variables, $|\psi|$ and $S[60,62,63,69-73]$. The model gives rise to classical-like analogy describing the motion of particles' density $n=|\psi|^{2}$ owning the 4-impulse $p_{\mu}=-\partial_{\mu} S$.

As shown by Weiner et al. [71], the outputs of the quantum hydrodynamic model agree with the outputs of the Schrödinger problem, but not only for the semi-classical limit or for a single 
particle [62,63,69]. More recently, Koide and Kodama [72], showed that it agrees with the outputs of the stochastic variational method.

Recently, the author of this article has shown that the hydrodynamic approach can be derived from the properties of vacuum on a small scale [73].

Moreover, as shown by Bohm and Hiley $[74,75]$ the hydrodynamic approach can be generalized for the description of the quantum fields.

The present work develops the quantum hydrodynamic form of the Klein-Gordon equation (KGE) containing an additional perturbative self-interaction term.

The interest of such a description lies in the fact that this kind of KGE can describe the states of bosons, such as mesons. The goal of this paper is to obtain the energy-impulse tensor of such a particle, for the coupling with the Einstein gravitational equation (GE) for quantum mechanical systems [68].

As shown in study [68], the quantum mechanical QG defines the gravitational coupling of classical fields and the commutation rules in curved space to be used for their quantization.

The paper is organized as follows: in Section 2, the hydrodynamic KGE with a perturbative self-interaction term is derived for scalar particle and for a charged boson. In Section 3, the formulae are generalized to a non-Euclidean space-time.

\section{The Hydrodynamic KGE with Perturbative Self-Interaction}

In this section, the Euclidean hydrodynamic representation of the KGE is derived for a scalar uncharged boson with a perturbative self-interaction term that reads

$$
\partial_{\mu} \partial^{\mu} \psi=-\frac{m^{2} c^{2}}{\hbar^{2}} \psi-\lambda|\psi|^{3}
$$

Following the procedure given in studies $[45,68]$ the hydrodynamic equations of motion are given by the Hamilton-Jacobi type equation

$$
g^{\mu \nu} \partial_{\mu} S \partial_{\nu} S-\hbar^{2}\left(\frac{\partial_{\mu} \partial^{\mu}|\psi|}{|\psi|}+\lambda|\psi|^{2}\right)-m^{2} c^{2}=0
$$

coupled with the current equation $[67,69]$

$$
\partial_{\mu}\left(|\psi|^{2} \partial_{\mu} S\right)=0
$$

where

$$
S=\frac{\hbar}{2 i} \ln \left[\frac{\psi}{\psi^{*}}\right]
$$

and where

$$
\frac{|\psi|^{2}}{m} \partial_{\mu} S=J_{\mu}=\frac{i \hbar}{2 m}\left(\psi^{*} \partial_{\mu} \psi-\psi \partial_{\mu} \psi^{*}\right)
$$

where the signature $(1,-1,-1,-1)$ has been used.

Moreover, being the 4-impulse of the hydrodynamic analogy

$$
p_{\mu}=-\partial_{\mu} S
$$

it follows that

$$
J_{\mu}=\left(c \rho,-J_{i}\right)=-|\psi|^{2} \frac{p_{\mu}}{m}
$$

where

$$
\rho=\frac{|\psi|^{2}}{m c^{2}} \frac{\partial S}{\partial t}
$$


Moreover, by using (6), Equation (2) can be rewritten as

$$
\partial_{\mu} S \partial^{\mu} S=p_{\mu} p^{\mu}=\left(\frac{E^{2}}{c^{2}}-p^{2}\right)=m^{2} c^{2}\left(1-\frac{V_{q u}}{m c^{2}}\right)
$$

where

$$
V_{q u}=-\frac{\hbar^{2}}{m}\left(\frac{\partial_{\mu} \partial^{\mu}|\psi|}{|\psi|}+\lambda|\psi|^{2}\right)
$$

and where $p^{2}=p_{i} p_{i}$ is the modulus of the spatial 3-impulse.

As shown in studies $[67,68]$, given the hydrodynamic Lagrangean function

$$
\begin{aligned}
L & =\frac{d S}{d t}=\frac{\partial S}{\partial t}+\frac{\partial S}{\partial q_{i}} \dot{q}_{i}=-p_{\mu} \dot{q}^{\mu} \\
& =\frac{1}{2} \frac{\sum_{n} a_{n}\left|\psi_{n}\right| \exp \left[\frac{i S_{n}}{\hbar}\right]\left(\frac{\hbar}{i} \dot{q}^{\mu} \partial_{\mu} l n\left|\psi_{n}\right|+L_{n}\right)}{\sum_{n} a_{n}\left|\psi_{n}\right| \exp \left[\frac{i S_{n}}{\hbar}\right]} \\
& -\frac{1}{2} \frac{\sum_{n} a^{*}{ }_{n}\left|\psi_{n}\right| \exp \left[\frac{i S_{n}}{\hbar}\right]\left(\frac{\hbar}{i} \dot{q}^{\mu} \partial_{\mu} l n\left|\psi_{n}\right|-L_{n}\right)}{\sum_{n} a^{*}{ }_{n}\left|\psi_{n}\right| \exp \left[\frac{-i S_{n}}{\hbar}\right]}
\end{aligned}
$$

Equation (2) can be expressed by the following system of Lagrangean equations of motion

$$
\begin{aligned}
& p_{\mu}=-\frac{\partial L}{\partial \dot{q}^{\mu}}, \\
& \dot{p}_{\mu}=-\frac{\partial L}{\partial q^{\mu}} .
\end{aligned}
$$

that for the eigenstates read

$$
\begin{aligned}
& p_{n_{\mu}}=-\frac{\partial L_{n}}{\partial \dot{q}^{\mu}} \\
& \dot{p}_{n_{\mu}}=-\frac{\partial L_{n}}{\partial q^{\mu}}
\end{aligned}
$$

where

$$
L_{n}=( \pm)-\frac{m c^{2}}{\gamma} \sqrt{1-\frac{V_{q u(n)}}{m c^{2}}}=( \pm)-\frac{m c^{2}}{\gamma} \sqrt{1+\frac{\hbar^{2}}{m^{2} c^{2}}\left(\frac{\partial_{\mu} \partial^{\mu}\left|\psi_{n}\right|}{\left|\psi_{n}\right|}+\lambda\left|\psi_{n}\right|^{2}\right)}
$$

where $c$ is the speed of light and $\gamma=\frac{1}{\sqrt{1-\frac{\dot{q}^{2}}{c^{2}}}}$. Generally speaking, for eigenstates, for which it holds $E=E_{n}=$ const, it follows that

$$
\begin{aligned}
& \left(\frac{E_{n}{ }^{2}}{c^{2}}-p_{n}{ }^{2}\right)=m^{2} c^{2}\left(1-\frac{V_{q u(n)}}{m c^{2}}\right) \\
& =m^{2} \gamma^{2} c^{2}\left(1-\frac{V_{q u(n)}}{m c^{2}}\right)-m^{2} \gamma^{2} \dot{q}^{2}\left(1-\frac{V_{q u(n)}}{m c^{2}}\right)
\end{aligned}
$$

from where it follows that

$$
E_{n}= \pm m \gamma c^{2} \sqrt{1-\frac{V_{q u(n)}}{m c^{2}}}= \pm m \gamma c^{2} \sqrt{1+\frac{\hbar^{2}}{m^{2} c^{2}}\left(\frac{\partial_{\mu} \partial^{\mu}\left|\psi_{n}\right|}{\left|\psi_{n}\right|}+\lambda\left|\psi_{n}\right|^{2}\right)}
$$

(where the minus sign stands for antiparticles) and, by using (17), that

$$
p_{n \mu}= \pm m \gamma \dot{q}_{\mu} \sqrt{1-\frac{V_{q u(n)}}{m c^{2}}}=\frac{E_{n}}{c^{2}} \dot{q}_{\mu}
$$


Following the hydrodynamic protocol [68], the eigenstates are represented by the stationary solutions of the hydrodynamic equations of motion obtained by deriving $p_{\mu_{(i, q)}}$ from (14) and then inserting it into (15) that leads to

$$
\begin{aligned}
\frac{d p_{n \mu}}{d s} & =-\frac{\gamma}{c} \frac{\partial L_{n}}{\partial q^{\mu}}= \pm \frac{d}{d s}\left(m c u_{\mu}\left(\sqrt{1-\frac{V_{q u)(n)}}{m c^{2}}}\right)\right) \\
& = \pm m c \frac{\partial}{\partial q^{\mu}} \sqrt{1-\frac{V_{q u(n)}}{m c^{2}}}
\end{aligned}
$$

where

$$
u_{\mu}=\frac{\gamma}{c} \dot{q}_{\mu^{\prime}}
$$

and to

$$
\pm m c \sqrt{1-\frac{V_{q u(n)}}{m c^{2}}} \frac{d u_{\mu}}{d s}=( \pm)-m c u_{\mu} \frac{d}{d s}\left(\sqrt{1-\frac{V_{q u(n)}}{m c^{2}}}\right) \pm m c \frac{\partial}{\partial q^{\mu}}\left(\sqrt{1-\frac{V_{q u(n)}}{m c^{2}}}\right)=-\frac{\gamma}{c} \frac{\partial \mathrm{T}_{n \mu^{v}}}{\partial q^{v}}
$$

where for eigenstates, the quantum energy-impulse tensor (QEIT) $\mathrm{T}_{n \mu}{ }^{v}$ reads $[45,68]$

$$
\mathrm{T}_{n \mu}{ }^{v}=\left(\dot{q}_{\mu} \frac{\partial L_{n}}{\partial \dot{q}_{v}}-L_{n} \delta_{\mu}{ }^{v}\right)= \pm \frac{m c^{2}}{\gamma} \sqrt{1-\frac{V_{q u(n)}}{m c^{2}}}\left(u_{\mu} u^{v}-\delta_{\mu}{ }^{v}\right) .
$$

leading to the quantum energy impulse tensor density (QEITD) $[45,68]$

$$
\mathrm{T}_{n \mu}{ }^{v}=\dot{q}_{\mu} \frac{\partial \boldsymbol{L}_{n n}}{\partial \dot{q}_{v}}-\boldsymbol{L}_{n n} \delta_{\mu}{ }^{v}=\left|\psi_{n}\right|^{2}\left(\dot{q}_{\mu} \frac{\partial L_{n}}{\partial \dot{q}_{v}}-L_{n} \delta_{\mu}{ }^{v}\right)=\left|\psi_{n}\right|^{2} \mathrm{~T}_{n \mu}{ }^{v}
$$

where $L=|\psi|^{2} L$ is the (hydrodynamic) Lagrangian density and $L$ is the hydrodynamic Lagrangian function.

The quantization condition is brought inside the above quantum hydrodynamic relations by the quantum potential $V_{q u(n)}$ contained in the term $\sqrt{1-\frac{V_{q u(n)}}{m c^{2}}}$. The analog quantities of the classical limit are recovered for $\hbar \rightarrow 0$, and the energy-impulse tensor (23) in Equation (22) leads to the classical equation of motion.

Moreover, by using the identity

$$
S_{n}=\frac{\hbar}{2 i} \ln \left[\frac{\psi_{n}}{\psi_{n}{ }^{*}}\right]
$$

the QEITD (24) can be written as a function of the wave function as following

$$
\begin{aligned}
T_{n \mu}{ }^{v}= & \pm|\psi|^{2} c^{2}\left(\frac{\partial S_{n}}{\partial t}\right)^{-1}\left(p_{\mu} p^{v}-p_{\alpha} p^{\alpha} \delta_{\mu}^{v}\right) \\
= & \pm m\left|\psi_{n}\right|^{2} c^{2}\left(\frac{\frac{\hbar}{2 m^{2} c^{2}} \partial \ln \left[\frac{\psi_{n}}{\psi_{n}{ }^{*}}\right]}{\partial t}\right)^{-1} \\
& \left(\left(\frac{\hbar}{2 m c}\right)^{2} \frac{\partial \ln \left[\frac{\psi_{n}}{\psi^{*} n}\right]}{\partial q^{\mu}} \frac{\partial \ln \left[\frac{\psi_{n}}{\psi_{n}}\right]}{\partial q_{\nu}}+\left(1-\frac{V_{q u(n)}}{m c^{2}}\right) \delta_{\mu}^{v}\right)
\end{aligned}
$$

\section{Charged Boson}

In the case of a charged boson, Equations (1)-(3) read, respectively,

$$
D_{\mu} D_{\mu} \psi=-\psi\left(\frac{m^{2} c^{2}}{\hbar^{2}}+\lambda|\psi|^{2}\right)
$$


where $D_{\mu}=\partial_{\mu}-\frac{e}{i \hbar} A_{\mu}$

$$
\begin{gathered}
\left(\partial_{\mu} S+e A_{\mu}\right)\left(\partial^{\mu} S+e A^{\mu}\right)=m^{2} c^{2}+\hbar^{2}\left(\frac{\partial_{\mu} \partial^{\mu}|\psi|}{|\psi|}+\lambda|\psi|^{2}\right) \\
\frac{\partial J_{\mu}}{\partial q_{\mu}}=0
\end{gathered}
$$

where the 4-current $J_{\mu}$ reads

$$
\begin{aligned}
J_{\mu} & =\left(c \rho,-J_{i}\right)=\frac{\hbar}{2 i m}\left(\psi^{*}\left(\partial_{\mu}-\frac{e}{i \hbar} A_{\mu}\right) \psi-\psi\left(\partial_{\mu}+\frac{e}{i \hbar} A_{\mu}\right) \psi^{*}\right) \\
& =-\frac{|\psi|^{2}}{m}\left[p_{\mu}-e A_{\mu}\right]=-\frac{|\psi|^{2}}{m} \pi_{\mu}
\end{aligned}
$$

and

$$
-\partial_{\mu} S=p_{\mu}=\pi_{\mu}+e A_{\mu}=\left(\frac{E}{c},-p_{i}\right)
$$

(where $\pi_{\mu}$ is the mechanical momentum) $[67,68]$ and where

$$
\rho=-\frac{|\psi|^{2}}{m c^{2}}\left[\frac{\partial S}{\partial t}+e \phi\right]
$$

Moreover, analogously to (9) and (17)-(19), from (28) it follows that

$$
\begin{aligned}
\pi_{n_{\mu}} & =\frac{1}{c^{2}}\left[-\frac{\partial S_{n}}{\partial t}-e \phi\right] \dot{q}_{\mu} \\
& =\frac{E_{n}-e \phi}{c^{2}} \dot{q}_{\mu}=p_{n_{\mu}}-e A_{\mu}
\end{aligned}
$$

that leads to

$$
E_{n}-e \phi= \pm m \gamma c^{2} \sqrt{1-\frac{V_{q u(n)}}{m c^{2}}}= \pm m \gamma c^{2} \sqrt{1+\frac{\hbar^{2}}{m^{2} c^{2}}\left(\frac{\partial_{\mu} \partial^{\mu}\left|\psi_{n}\right|}{\left|\psi_{n}\right|}+\lambda\left|\psi_{n}\right|^{2}\right)},
$$

to

$$
p_{n \mu}-e A_{\mu}= \pm m \gamma \dot{q}_{\mu} \sqrt{1-\frac{V_{q u(n)}}{m c^{2}}}=\frac{E_{n}}{c^{2}} \dot{q}_{\mu}
$$

and to

$$
\begin{aligned}
L_{n} & =\frac{d S_{n}}{d t}=\frac{\partial S_{n}}{\partial t}+\frac{\partial S_{n}}{\partial q_{i}} \dot{q}_{i}=-p_{n \mu} \dot{q}^{\mu}=-\left(\frac{1}{c^{2}} \frac{\partial S_{n}}{\partial t}+\frac{e}{c^{2}} \phi\right)^{-1} p_{n \mu}\left(p_{n}{ }^{\mu}-e A^{\mu}\right) \\
& =( \pm)\left(-\frac{m c^{2}}{\gamma} \sqrt{1+\frac{\hbar^{2}}{m^{2} c^{2}}\left(\frac{\partial \partial_{\mu} \partial^{\mu}\left|\psi_{n}\right|}{\left|\psi_{n}\right|}+\lambda\left|\psi_{n}\right|^{2}\right)}-e A_{\mu} \dot{q}^{\mu}\right)
\end{aligned}
$$

that by using (25), as a function of $\psi$ and $A^{\mu}$, reads

$$
L_{n}=-\frac{i \hbar}{2} c^{2}\left(\frac{\partial \ln \left[\frac{\psi_{n}}{\psi_{n} *}\right]}{\partial t}+\frac{2 i e}{\hbar} \phi\right)^{-1} \frac{\partial \ln \left[\frac{\psi_{n}}{\psi_{n} *}\right]}{\partial q^{\mu}}\left(\frac{\partial \ln \left[\frac{\psi_{n}}{\psi_{n} *}\right]}{\partial q_{\mu}}-\frac{2 i e}{\hbar} A^{\mu}\right) .
$$

For $\hbar=0$ the Lagrangean (37) acquires the known classical expression $L=-\left(\frac{m c^{2}}{\gamma}+e A_{\mu} \dot{q}^{\mu}\right)$. Moreover, with the help of (25), (30), (33)-(35) it follows that

$$
T_{n \mu^{v}}{ }^{v}=( \pm)-\frac{m\left|\psi_{n}\right|^{2} c^{2}}{\gamma} \sqrt{1-\frac{V_{q u(n)}}{m c^{2}}}\left(\begin{array}{c}
\left(u_{\mu}+\left(\sqrt{1-\frac{V_{q u(n)}}{m c^{2}}}\right)^{-1} \frac{e}{m c} A_{\mu}\right) u^{v} \\
-\left(1+\left(\sqrt{1-\frac{V_{q u(n)}}{m c^{2}}}\right)^{-1} \frac{e}{m c} A_{\alpha} u^{\alpha}\right) \delta_{\mu}{ }^{v}
\end{array}\right)
$$


that by using (25), (30) and (35) we can be expressed as a function of the wave function as

$$
T_{\mu}^{v}=\left|\psi_{n}\right|^{2} \frac{\hbar c^{2}}{2 i}\left(\frac{\partial}{\partial t} \ln \left[\frac{\psi_{n}}{\psi_{n}{ }^{*}}\right]-\frac{2 i e}{\hbar} \phi\right)^{-1}\left(\begin{array}{c}
\left(\frac{\partial \ln \left[\frac{\psi_{n}}{\psi^{*}}\right]}{\partial q^{*}}-\frac{2 i e}{\hbar} A_{\mu}\right) \frac{\partial \ln \left[\frac{\psi_{n}}{\psi_{n}}\right]}{\partial q_{\nu}} \\
-\left(\frac{\partial \ln \left[\frac{\psi_{n}}{\left.\psi_{n^{*}}\right]}\right.}{\partial q^{\alpha}}-\frac{2 i e}{\hbar} A_{\alpha}\right) \frac{\partial \ln \left[\frac{\psi_{n}}{\left.\psi_{n}\right]}\right]}{\partial q_{\alpha}} \delta_{\mu}{ }^{v}
\end{array}\right)
$$

The above equations are coupled to the Maxwell equation:

$$
F_{; v}^{\mu v}=-4 \pi J^{\mu}
$$

where [75]

$$
F_{\mu \nu}=\left(A_{v, \mu}-A_{\mu, v}\right)=\left(\partial_{\mu} A_{\nu}-\partial_{\nu} A_{\mu}\right),
$$

and where

$$
A_{\mu}=\left(\frac{\phi}{c},-A_{i}\right)
$$

is the potential 4-vector.

\section{Non-Euclidean Space-Time}

The quartic self-interaction is introduced in the KGE in order to describe the states of charged $( \pm 1)$ bosons (e.g., mesons) [76]. The importance of having the hydrodynamic description of bosons [68] lies in the fact that it becomes possible to derive its quantum energy-impulse tensor that can couple them to the Einstein gravitational equation for quantum mechanical systems [68].

By using the General Physics Covariance postulate [3,68], it is possible to derive the non-Euclidean expression of the hydrodynamic model of the KGE:

$$
\left(\partial^{\mu} \psi\right)_{, \mu}=\frac{1}{\sqrt{-g}} \partial_{\mu}\left(\sqrt{-g} g^{\mu v} \partial_{\nu} \psi\right)=-\psi\left(\frac{m^{2} c^{2}}{\hbar^{2}}+\lambda|\psi|^{2}\right)
$$

Equations (2) and (3) in a non-Euclidean space-time read, respectively,

$$
\begin{aligned}
& g^{\mu v} \partial_{\mu} S \partial_{\nu} S+m V_{q u}-m^{2} c^{2}=0 \\
& \frac{1}{\sqrt{-g}} \partial_{\mu} \sqrt{-g}\left(g^{\mu v}|\psi|^{2} \partial_{\nu} S\right)=0
\end{aligned}
$$

where

$$
V_{q u}=-\frac{\hbar^{2}}{m}\left(\frac{1}{|\psi| \sqrt{-g}} \partial_{\mu} \sqrt{-g}\left(g^{\mu v} \partial_{v}|\psi|\right)+\lambda|\psi|^{2}\right) .
$$

Moreover, by using the Lagrangean function

$$
L_{n}=\frac{d S_{n}}{d t}=-g_{\mu v} p_{n}{ }^{v} \dot{q}^{\mu}
$$

the covariant form of the motion Equations (14) and (15) reads

$$
\begin{gathered}
p_{n_{\mu}}=-\frac{\partial L_{n}}{\partial \dot{q}^{\mu}} \\
D_{t} p_{n_{\mu}}=-\frac{\partial L_{n}}{\partial q^{\mu}}
\end{gathered}
$$

where

$$
D_{t} p_{\mu}=\dot{p}_{\mu}-\Gamma_{\mu \lambda}^{v} p_{v} \dot{q}^{\lambda}
$$


is the total covariant derivative respect the time and where $\Gamma_{\mu \lambda}^{v}$ are the Christoffel symbols.

Equations (47) and (48) leads to the motion equation

$$
D_{t}\left(-\frac{\partial L_{n}}{\partial \dot{q}^{\mu}}\right)=-\frac{\partial L_{n}}{\partial q^{\mu}}
$$

where $L_{n}$ reads

$$
L_{n}=( \pm)-\frac{m c^{2}}{\gamma} \sqrt{1-\frac{V_{q u(n)}}{m c^{2}}}=( \pm)-m c^{2} \sqrt{\frac{g_{\mu v} \dot{q}^{v} \dot{q}^{\mu}}{c^{2}}} \sqrt{1-\frac{V_{q u(n)}}{m c^{2}}} .
$$

From (50) it follows that the motion equation, describing the evolution of the particle density $n=\left|\psi_{n}\right|^{2}$ moving with the hydrodynamic impulse (6), reads

$$
\frac{d u_{\mu}}{d t}-\frac{1}{2} \frac{c}{\gamma} \frac{\partial g_{\lambda \kappa}}{\partial q^{\mu}} u^{\lambda} u^{\kappa}=\frac{c}{\gamma} \frac{\partial \ln \sqrt{1-\frac{V_{q u(n)}}{m c^{2}}}}{\partial q^{\mu}}+\frac{c}{\gamma} \frac{\partial \ln \gamma}{\partial q^{\mu}}-u_{\mu} \frac{d}{d t} \ln \sqrt{1-\frac{V_{q u}}{m c^{2}}}
$$

where the stationary condition $\frac{d u_{\mu}}{d t}=0$, that determines the balance between the "force" of gravity and that one of the quantum potential, defines the stationary equation for the eigenstates

$$
\frac{1}{2} \frac{c}{\gamma} \partial_{\mu} g_{\lambda \kappa} u^{\lambda} u^{\kappa}+\frac{c}{\gamma} \partial_{\mu} \ln \gamma=-\frac{c}{\gamma} \partial_{\mu} \ln \sqrt{1-\frac{V_{q u(n)}}{m c^{2}}}+u_{\mu} \frac{d}{d t} \ln \sqrt{1-\frac{V_{q u}}{m c^{2}}} .
$$

where $\frac{1}{g}=\left|g_{v \mu}\right|=-J_{a c}{ }^{2}$, where $J_{a c}$ is Jacobean of the transformation of the Galilean co-ordinates to non-Euclidean ones and where $g_{v \mu}$ is the metric tensor defined by the gravitational equation for quantum mechanical systems $[68,75]$

$$
R_{\mu v}-\frac{1}{2} g_{\mu v} R_{\alpha}{ }^{\alpha}-\Lambda g_{\mu v}=\frac{8 \pi G}{c^{4}} T_{\mu v}
$$

where the quantum energy impulse tensor density reads

$$
T_{(n) \mu v}= \pm\left|\psi_{n}\right|^{2} m c^{2} \sqrt{\frac{g_{\mu \nu} \dot{q}^{v} \dot{q}^{\mu}}{c^{2}}} \sqrt{1-\frac{V_{q u(n)}}{m c^{2}}}\left(u_{\mu} u_{v}-g_{\alpha \beta} u^{\alpha} u^{\beta} g_{\mu v}\right) .
$$

And where the cosmological energy-impulse density $\Lambda$ [68], for eigenstates, reads

$$
\Lambda=\frac{8 \pi G}{c^{4}}\left|\psi_{n}\right|^{2} L_{0}=\frac{8 \pi G}{c^{4}} \frac{m\left|\psi_{n}\right|^{2} c^{2}}{\gamma}
$$

where for scalar uncharged particles leads to

$$
L_{0}=\lim _{\hbar \rightarrow 0} L=( \pm)-\frac{m c^{2}}{\gamma} .
$$

The quantum energy impulse tensor density for the particle density distribution $\left|\psi_{n}\right|^{2}$ owing with the hydrodynamic impulse (6), as a function of the wave function reads

$$
\begin{aligned}
T_{\mu v}=T_{\mu}{ }^{\alpha} g_{\alpha \nu} & =|\psi|^{2} m^{2} c^{4}\left(\frac{\partial S}{\partial t}\right)^{-1}\left(\frac{p_{\mu} p_{v}}{m^{2} c^{2}}-\left(1-\frac{V_{q u}}{m c^{2}}\right) g_{\mu v}\right) \\
& =m|\psi|^{2} c^{2}\left(\frac{\frac{\hbar}{2 i m^{2} c^{2}} \partial \ln \left[\frac{\psi}{\partial *}\right]}{\partial t}\right)^{-1} \\
& \left(\left(\frac{\hbar}{2 m c}\right)^{2} \frac{\partial \ln \left[\frac{\psi}{\psi *}\right]}{\partial q^{\mu}} \frac{\partial \ln \left[\frac{\psi}{\psi^{*}}\right]}{\partial q^{v}}+\left(1-\frac{V_{q u}}{m c^{2}}\right) g_{\mu v}\right)
\end{aligned}
$$


Charged Boson in Non-Euclidean Space-Time

The KGE in non-Euclidean space-time for electromagnetic charged boson

$$
\frac{1}{\sqrt{-g}} D^{\mu}\left(\sqrt{-g} D_{\mu} \psi\right)-\frac{A_{\mu} \partial^{\mu} \sqrt{-g}}{\sqrt{-g}}=-\psi\left(\frac{m^{2} c^{2}}{\hbar^{2}}+\lambda|\psi|^{2}\right)
$$

leads to the hydrodynamic system of equations

$$
\begin{gathered}
\frac{1}{\sqrt{-g}} \partial_{\mu} \sqrt{-g}\left(g^{\mu v}|\psi|^{2}\left(\partial_{v}+e A_{v}\right)\right)=0 \\
g^{\mu v}\left(\partial_{\mu} S+e A_{\mu}\right)\left(\partial_{\nu} S+e A_{v}\right)=m^{2} c^{2}-m V_{q u}
\end{gathered}
$$

where

$$
V_{q u}=-\frac{\hbar^{2}}{m}\left(\frac{1}{|\psi| \sqrt{-g}} \partial_{\mu} \sqrt{-g}\left(g^{\mu v} \partial_{\nu}|\psi|\right)+\lambda|\psi|^{2}\right),
$$

Moreover, the Lagrangean motion equations read

$$
\begin{gathered}
p_{n_{\mu}}=-\frac{\partial L_{n}}{\partial \dot{q}^{\mu}} \\
D_{t} p_{n_{\mu}}=-\frac{\partial L_{n}}{\partial q^{\mu}},
\end{gathered}
$$

where

$$
L_{n}=( \pm)\left(-m c^{2} \sqrt{\frac{g_{\mu \nu} \dot{q}^{\nu} \dot{q}^{\mu}}{c^{2}}} \sqrt{1+\frac{\hbar^{2}}{m^{2} c^{2}}\left(\frac{\partial_{\mu} \partial^{\mu}\left|\psi_{n}\right|}{\left|\psi_{n}\right|}+\lambda\left|\psi_{n}\right|^{2}\right)}-g_{\mu \nu} e A^{v} \dot{q}^{\mu}\right)
$$

and to the QIETD

$$
\begin{aligned}
T_{n \mu^{v}}= & ( \pm)-m\left|\psi_{n}\right|^{2} c^{2} \sqrt{\frac{g_{\mu \nu} \dot{q}^{v} \dot{q}^{\mu}}{c^{2}}} \sqrt{1-\frac{V_{q u(n)}}{m c^{2}}} \\
& \left(\begin{array}{l}
\left(u_{\mu}+\left(\sqrt{1-\frac{V_{q u(n)}}{m c^{2}}}\right)^{-1} \frac{e}{m c} A_{\mu}\right) u^{v} \\
-\left(1+\left(\sqrt{1-\frac{V_{q u(n)}}{m c^{2}}}\right)^{-1} \frac{e}{m c} g_{\alpha \beta} A^{\beta} u^{\alpha}\right) \delta_{\mu}{ }^{v}
\end{array}\right)
\end{aligned}
$$

\section{Discussion and Conclusions}

Even if the GE for quantum systems (54) is formally equal to the Einstein GE, it actually demonstrates some differences. If the Einstein GE refers to the gravitational description of classical masses with no information, either about the quantum mechanical properties of bodies or about the string properties of elementary particles building up the matter, the GE (54) introduces the quantum properties in the gravity of a mass distribution that leads to a cosmological term, that is not a simple constant, but rather, has the form of an energy-impulse tensor density that leads to the correct value of the cosmological constant on the galactic space for the description of the motion of galaxies [68].

Even if the gravity of a quantum mechanical system refers to non-quantized fields, it is not without interest, since it is able to define the gravitational coupling of classical fields and their commutation rules in the non Euclidean space-time for the derivation of the quantum gravity equation of quantum fields.

The GE (54) together with (53) allows also to derive the mass eigenvalues of a Schwarzschild black hole $[45,68]$ (like the e-atom model that is currently of interest to the scientific community [38] (and references therein)). 
Finally, in this work the hydrodynamic model for bosons described by a KGE with perturbative self-interacting field is derived for defining the coupling with the Einstein gravitational equation of quantum mechanical systems.

The hydrodynamic treatment has a classical-like structure where the quantum dynamics (quantization) is introduced by the quantum potential through the factor $\sqrt{1-\frac{V_{q u(n)}}{m c^{2}}}$.

This can be clearly seen by the Lagrangean function $L_{n}=( \pm)-\frac{m c^{2}}{\gamma} \sqrt{1-\frac{V_{q u(n)}}{m c^{2}}}=( \pm)-$ $m c^{2} \sqrt{\frac{g_{\mu v} \dot{q}^{v} \dot{q}^{\mu}}{c^{2}}} \sqrt{1-\frac{V_{q u(n)}}{m c^{2}}}$ that, in the classical limit for $\hbar \rightarrow 0$ (i.e., $V_{q u(n)}=0$ and $\sqrt{1-\frac{V_{q u(n)}}{m c^{2}}}=1$ ), leads to the known classical expression.

Given the classical-like hydrodynamic model of quantum mechanics, the energy-impulse tensor for the Einstein gravitational equation of quantum mechanical systems is straightforwardly derived. The availability of such a description for mesons facilitates the coupling of them to the GE.

The biunique correspondence between the standard quantum mechanics and the hydrodynamic representation [60-63,70,71,77] warrants that the quantum energy-impulse tensor density is independent by the used formalism.

Conflicts of Interest: The author declares no conflict of interest.

\section{References}

1. Rovelli, C. Quantum Gravity; Cambridge University Press: Cambridge, UK, 2004.

2. Bousso, R. The Holographic principle. Rev. Mod. Phys. 2002, 74, 825. [CrossRef]

3. Ashtekar, A. Introduction to loop quantum gravity and cosmology. In Quantum gravity and quantum cosmology; Springer: Berlin, Germany, 2013; pp. 31-56.

4. Rovelli, C.; Vidotto, F. Covariant Loop Quantum Gravity; Cambridge University Press: Cambridge, UK, 2014.

5. Thiemann, T. Modern Canonical Quantum General Relativity; Cambridge University Press: Cambridge, UK, 2007.

6. Giddings, S.B. Is string theory a theory of quantum gravity? Found. Phys. 2013, 43, 115-139. [CrossRef]

7. Dewitt, B.S. Quantum theory of gravity. I. The canonical theory. Phys. Rev. 1967, 160, 1113. [CrossRef]

8. Gupta, S.N. Quantization of Einstein's gravitational field: General treatment. Proc. Phys. Soc. Sect. A 1952, 65, 608-619. [CrossRef]

9. Gupta, S.N.; Radford, S.F. Quantum field-theoretical electromagnetic and gravitational two-particle potentials. Phys. Rev. D 1980, 21, 2213. [CrossRef]

10. Faddeev, L.D.; Popov, V.N. Feynman diagrams for the Yang-Mills field. Phys. Lett. B 1967, 25, 29-30. [CrossRef]

11. Mandelstam, S. Feynman rules for the gravitational field from the coordinate-independent field-theoretic formalism. Phys. Rev. 1968, 175, 1604. [CrossRef]

12. Schwinger, J. Quantized gravitational field. Phys. Rev. 1963, 130, 1253. [CrossRef]

13. Schwinger, J. Sources and gravitons. Phys. Rev. 1968, 173, 1264-1272. [CrossRef]

14. Weinberg, S. Photons and gravitons in S-matrix theory: Derivation of charge conservation and equality of gravitational and inertial mass. Phys. Rev. B 1964, 135, B1049. [CrossRef]

15. Iwasaki, Y. Quantum theory of gravitation vs. classical theory fourth-order potential. Prog. Theor. Phys. 1971, 46, 1587-1609. [CrossRef]

16. T Hooft, G.; Veltman, M.J.G. One-loop divergencies in the theory of gravitation. Ann. Henri Poincaré 1974, 20, 69-94.

17. Radkowski, A.F. Some aspects of the source description of gravitation. Ann. Phys. 1970, 56, 319-354. [CrossRef]

18. Capper, D.M.; Leibbrandt, G.; Ramon Medrano, M. Calculation of the graviton self-energy using dimensional regularization. Phys. Rev. D 1973, 8, 4320. [CrossRef]

19. Duff, M.J. Quantum corrections to the Schwarzschild solution. Phys. Rev. D 1974, 9, 1837. [CrossRef]

20. Capper, D.M.; Duff, M.J.; Halpern, L. Photon corrections to the graviton propagator. Phys. Rev. D 1974, 10, 461. [CrossRef] 
21. Capper, D.M.; Duff, M.J. The one loop neutrino contribution to the graviton propagator. Nucl. Phys. B 1974, 82, 147-154. [CrossRef]

22. Duff, M.J.; Liu, J.T. Complementarity of the Maldacena and Randall-Sundrum pictures. Phys. Rev. Lett. 2000, 85, 2052. [CrossRef] [PubMed]

23. Odintsov, S.D.; Shapiro, I.L. General relativity as the low-energy limit in higher derivative quantum gravity. Class. Quantum Gravity 1992, 9, 873. [CrossRef]

24. Elizalde, E.; Odintsov, S.D.; Shapiro, I.L. Asymptotic regimes in quantum gravity at large distances and running Newtonian and cosmological constants. Class. Quantum Gravity 1994, 11, 1607. [CrossRef]

25. Elizalde, E.; Lousto, C.O.; Odintsov, S.D.; Romeo, A. GUT's in curved spacetime: Running gravitational constants, Newtonian potential, and the quantum-corrected gravitational equations. Phys. Rev. D 1995, 52, 2202. [CrossRef]

26. Dalvit, D.A.R.; Mazzitelli, F.D. Running coupling constants, Newtonian potential, and nonlocalities in the effective action. Phys. Rev. D 1994, 50, 1001. [CrossRef]

27. Dalvit, D.A.R.; Mazzitelli, F.D. Geodesics, gravitons, and the gauge-fixing problem. Phys. Rev. D 1997, 56, 7779. [CrossRef]

28. Satz, A.; Mazzitelli, F.D.; Alvarez, E. Vacuum polarization around stars: Nonlocal approximation. Phys. Rev. D 2005, 71, 064001. [CrossRef]

29. Anderson, P.R.; Fabbri, A. Apparent universality of semiclassical gravity in the far field limit. Phys. Rev. D 2007, 75, 044015. [CrossRef]

30. Stephens, C.R.; Hooft, G.t.; Whiting, B.F. Black hole evaporation without information loss. Class. Quantum Gravity 1994, 11, 621. [CrossRef]

31. Hawking, S.W. Particle creation by black holes. Commun. Math. Phys. 1975, 43, 199-220. [CrossRef]

32. Parikh, M.K.; Wilczek, F. Hawking radiation as tunneling. Phys. Rev. Lett. 2000, 85, 5042-5045. [CrossRef] [PubMed]

33. Parikh, M.K. A secret tunnel through the horizon. Gen. Relat. Gravitat. 2004, 36, 2419-2422. [CrossRef]

34. Banerjee, R.; Majhi, B.R. Quantum tunneling beyond semiclassical approximation. J. High Energy Phys. 2008, 2008, 095. [CrossRef]

35. Angheben, M.; Nadalini, M.; Vanzo, L.; Zerbini, S. Hawking radiation as tunneling for extremal and rotating black holes. J. High Energy Phys. 2005, 2005, 014. [CrossRef]

36. Arzano, M.; Medved, A.J.M.; Vagenas, E.C. Hawking radiation as tunneling through the quantum horizon. J. High Energy Phys. 2005, 2005, 037. [CrossRef]

37. Banerjee, R.; Majhi, B.R. Hawking black body spectrum from tunneling mechanism. Phys. Lett. B 2009, 675, 243-245. [CrossRef]

38. Corda, C. Quasi-normal modes: The electrons of black holes as gravitational atoms? Implications for the black hole information puzzle. Adv. High Energy Phys. 2015, 2015, 867601. [CrossRef]

39. Hajcek, P.; Kiefer, C. Singularity avoidance by collapsing shells in quantum gravity. Int. J. Mod. Phys. D 2001, 10, 775-779. [CrossRef]

40. Bambi, C.; Malafarina, D.; Modesto, L. Non-singular quantum-inspired gravitational collapse. Phys. Rev. D 2013, 88, 044009. [CrossRef]

41. Rovelli, C.; Vidotto, F. Planck stars. Int. J. Mod. Phys. D 2014, 23, 1442026. [CrossRef]

42. Barrau, A.; Rovelli, C. Planck star phenomenology. Phys. Lett. B 2014, 739, 405-409. [CrossRef]

43. Carr, B.J.; Mureika, J.; Nicolini, P. Sub-Planckian black holes and the Generalized Uncertainty Principle. J. High Energy Phys. 2015, 2015, 1-24. [CrossRef]

44. T Hooft, G. The quantum black hole as a hydrogen atom: Microstates without strings attached. arXiv, 2016.

45. Chiarelli, P. The quantum lowest limit to the black hole mass. Phys. Sci. Int. J. 2016, 9, 1-25. [CrossRef]

46. Einstein, A. Zum Kosmologischen Problem der Allgemeinen Relativit Atstheorie; Wiley-VCH Verlag GmbH \& Co. KGaA: Weinheim, Germany, 1931; Volume 142, pp. 235-237.

47. Carroll, S.M.; Press, W.H.; Turner, E.L. The cosmological constant. Annu. Rev. Astron. Astrophys. 1992, 30, 499-542. [CrossRef]

48. Zel'dovich, Y.B. The cosmological constant and the theory of elementary. Sov. Phys. Uspekhi 1968, 11, 381-393. [CrossRef]

49. Gamov, G. My World Line; Viking Press: New York, NY, USA, 1970; p. 44. 
50. Hubble, E.P. A Relation between Distance and Radial Velocity among Extra-Galactic Nebulae. Proc. Natl. Acad. Sci. USA 1929, 15, 168-173. [CrossRef] [PubMed]

51. Cohn, J.D. Living with Lambda. Astrophys. Space Sci. 1998, 259, 213-234. [CrossRef]

52. Sahni, V.; Starobinsky, A.A. The case for a positive cosmological $\Lambda$-Term. Int. J. Mod. Phys. D 2000, 9, $373-443$. [CrossRef]

53. Turner, M.S. Dark matter and dark energy in the universe. In The Third Stromlo Symposium: The Galactic Halo, Proceedings of the Third Stromlo Symposium, Canberra, ACT, Australia, 17-21 August 1998; Gibson, B.K., Axelrod, T.S., Putman, M.E., Eds.; ASP Conference Series; ASP: San Francisco, CA, USA, 1998; Volume 165, pp. 431-452.

54. Weinberg, S. The cosmological constant problem. Rev. Mod. Phys. 1989, 61, 1-23. [CrossRef]

55. Dewitt, B.S. Quantum theory of gravity. II. The manifestly covariant theory. Phys. Rev. 1967, $162,1195$. [CrossRef]

56. Veltman, M. Gravitation, in Les Houches; Balian, R., Zinn-Justin, J., Eds.; Session XXVIII; North-Holland Publishing Company: Amsterdam, the Netherlands, 1976; p. 266.

57. Goroff, M.H.; Sagnotti, A. The ultraviolet behavior of Einstein gravity. Nucl. Phys. B 1986, 266, 709-736. [CrossRef]

58. Van de Ven, A.E. Two-loop quantum gravity. Nucl. Phys. B 1992, 378, 309-366. [CrossRef]

59. Akhundov, A.; Shiekh, A. A Review of leading quantum gravitational corrections to Newtonian gravity. arXiv 2006, arXiv:gr-qc/0611091.

60. Bialyniki-Birula, I.; Cieplak, M.; Kaminski, J. Theory of Quanta; Oxford University Press: New York, NY, USA, 1992; pp. 87-115.

61. Bohm, D. A suggested interpretation of the quantum theory in terms of "hidden" variables. I. Phys Rev. 1952, 85, 166. [CrossRef]

62. Madelung, E.Z. Quantentheorie in hydrodynamischer Form. Z. Physik A 1926, 40, 322-326. [CrossRef]

63. Jánossy, L. Zum hydrodynamischen Modell der Quantenmechanik. Z. Phys. 1962, 169, 79-89. [CrossRef]

64. Aharonov, Y.; Bohm, D. Significance of electromagnetic potentials in quantum theory. Phys. Rev. 1959, 115, 485-491. [CrossRef]

65. Weiner, J.H. Statistical Mechanics of Elasticity; John Wiley \& Sons: New York, NY, USA, 1983; pp. $315-317$.

66. Haas, F.; Eliasson, B.; Shukla, P.K. Relativistic Klein-Gordon-Maxwell multistream model for quantum plasmas. Phys. Rev. E 2012, 85, 056411. [CrossRef] [PubMed]

67. Chiarelli, P. The CPT-Ricci scalar curvature symmetry in quantum electro-gravity. Int. J. Sci. 2016, 5, 36-58. [CrossRef]

68. Chiarelli, P. The Quantum-Gravity Equation Derived from the Minimum Action Principle, Submitted for Publication. Available online: https: / / www.researchgate.net/publication/318420781_The_quantumgravity_equation_derived_from_the_minimum_action_principle (accessed on 1 September 2017).

69. Guvenis, H. Hydrodynamische Formulierung der relativischen Quantenmechanik. Available online: http: / / gsjournal.net/Science-Journals / Essays/View/5241 (accessed on 9 January 2014).

70. Chiarelli, P. Theoretical derivation of the cosmological constant in the framework of the hydrodynamic model of quantum gravity: The solution of the quantum vacuum catastrophe? Galaxies 2016, 4, 6. [CrossRef]

71. Weiner, J.H.; Askar, A. Particle method for the numerical solution of the time-dependent schrödinger equation. J. Chem. Phys. 1971, 54, 3534-3541. [CrossRef]

72. Koide, T.; Kodama, T. Stochastic variational method as quantization scheme: Field quantization of complex Klein-Gordon equation. Prog. Theor. Exp. Phys. 2015. [CrossRef]

73. Chiarelli, P. The Planck law for particles with rest mass. Quantum Matter 2016, 5, 748-751. [CrossRef]

74. Hiley, B.J.; Callaghan, R.E. The Clifford Algebra approach to Quantum Mechanics A: The Schrödinger and Pauli Particles. arXiv, 2010.

75. Landau, L.D.; Lifsits, E.M. Course of Theoretical Physics; Butterworth-Heinemann: Oxford, UK, 1976; Volume 2, pp. 309-335. 
76. Le Bellac, M. Quantum and Statistical Field Theory; Oxford Science Publication: Oxford, UK, 1991; pp. $315-337$.

77. Tsekov, R. Bohmian Mechanics versus Madelung Quantum Hydrodynamics. arXiv, 2009.

(c)

(C) 2017 by the author. Licensee MDPI, Basel, Switzerland. This article is an open access article distributed under the terms and conditions of the Creative Commons Attribution (CC BY) license (http:/ / creativecommons.org/licenses/by/4.0/). 